\title{
Uso y acceso a las TIC en estudiantes de escuelas secundarias públicas en la ciudad de Chihuahua, México: inclusión en la didáctica y en la alfabetización digital
}

\section{Use and access to ICT in students of public secondary schools in Chihuahua City, Mexico: inclusion in teaching and digital literacy}

\author{
Javier TARANGO, José Refugio ROMO-GONZÁLEZ, \\ Laura Patricia MURGUÍA-JÁQUEZ, Gerardo ASCENCIO-BACA \\ Universidad Autónoma de Chihuahua, México
}

Recibido: Enero 2013

Aceptado: Abril 2013

\section{Resumen}

El presente artículo aborda la cuestión de la brecha digital en estudiantes de escuelas secundarias públicas de la ciudad de Chihuahua, México, en razón de identificar la desigualdad de posibilidades que observan los sujetos estudiados para acceder a la información, conocimiento y educación por medio de las TIC (internet, telefonía móvil, banda ancha y televisión). El estudio toma como escenario investigativo tres escuelas, utilizando la encuesta como instrumento de recolección de datos, identificando patrones de comportamiento en cuanto a: conocimiento generalizado de las mismas, acceso a equipo de cómputo e internet y caracterización de su uso. Otros aspectos de análisis es la identificación del nivel educativo de los padres de familia y el acceso a los recursos tecnológicos disponibles con fines académicos y no académicos en diversos ámbitos de aplicación (hogar, escuela y entorno social). La propuesta concluye, a través de la recopilación de alternativas sugeridas por los docentes para incorporar las TIC con fines didácticos de forma sistemática y planeada, cuyo mayor reflejo se manifieste en mejores indicadores en alfabetización digital.

Palabras clave: herramientas para la evaluación de la alfabetización informacional, alfabetización informacional en estudiantes de escuelas secundarias públicas, alfabetización informacional y curriculum.

\footnotetext{
Abstract

This paper addresses the issue of the digital divide in students of public secondary schools at Chihuahua City, Mexico. It seeks to identify potential inequality of opportunities with regards to subjects' access to information, knowledge and education through the ICT (internet, mobile 
telephony, broadband and television). The study takes three schools as investigative stage, using the survey as a data collection instrument, identifying patterns of behavior regarding: general knowledge of them, access to computer equipment and internet, and characterization of their use. Other aspects of analysis are the identification of the educational level of parents and access to technology resources available for academic and non-academic purposes in various application areas (home, school and social environment). The proposal concludes, that it is through the recollection of alternatives suggested by the teachers themselves to incorporate ICT for teaching purposes in a systematic and planned fashion, whose greatest reflection manifests in better digital literacy indicators.

Keywords: tools for assessing information literacy, information literacy in public high school students, information literacy and curriculum.

La educación como la economía está inmersa en los intereses de los procesos productivos actuales, compuestos por una compleja red de elementos entrecruzados, en un mercado construido sobre bases universales. Para ello, se buscado cierta segmentación en los países de acuerdo a las condiciones más adecuadas, saltando por encima de las viejas fronteras inútiles (ahora eliminadas por la tecnología). La propuesta es que la educación sea creativa, cooperativa, independiente y con espíritu científico (Ramentol, 2004).

Tomando en consideración la premisa anterior, el presente estudio aborda a la brecha digital como un elemento fundamental en el desarrollo de los procesos educativos, considerando que la incorporación de las Tecnologías de Información y Comunicación (TIC) en el uso cotidiano de los estudiantes, puede convertirse en un fenómeno básico de cambio de paradigma que afectará positivamente sobre la manera de abordar los contenidos, siendo esta una preocupación constante de los docentes en todos los niveles educativos. Dentro de la educación básica en México (primaria y secundaria) ha sido un efecto del uso voluntario de los estudiantes que ha forzado la incorporación de medios electrónicos como obligatorios en sectores educativos de mayor nivel socioeconómico (especialmente en educación privada).

La presencia de las TIC en la educación ha pasado incluso del pensar del docente a una delimitación poco precisa de políticas públicas de los gobiernos en México, considerando para ello además del uso, la identificación de la forma como se emplea y las acciones que se generan a partir de que el sujeto emprenda acciones que favorezcan $\mathrm{su}$ aprovechamiento escolar. Las propuestas gubernamentales se han centrado en la posibilidad de identificar, primero, los niveles de brecha digital como un fenómeno natural del avance educativo $\mathrm{y}$, aunque pareciera secundario, buscar que los resultados se proyecten en indicadores internacionales de evaluación macrosocial, tales como los estudios realizados por la Organización para la Cooperación y el Desarrollo Económico (OCDE) por medio del examen PISA, cuyos resultados no han favorecido a México en comparación con otros países participantes, ya no se diga con Nueva Zelanda, Corea del Sur o España, sino con los propios países latinoamericanos.

El uso y manejo de las TIC como elemento educativo tiene una estrecha relación con la medición de la brecha digital. Este término es definido quizá de muchas maneras, sin embargo el más tangible es en relación con el acceso a computadoras e 
internet, así como en las habilidades de uso de esas tecnologías (UNESCO, 2009). Además, la tecnología se considera un proceso social, por lo que se demanda explorar un significado más amplio que haga referencia al contexto socioeconómico en donde se emplea, considerando para ello la forma como se usa y para qué se usa, siendo su nivel más alto cuando se identifican las contribuciones que esta clase de herramientas puede llegan a tener en la innovación social

El estudio de la brecha digital y las TIC en educación muestran una dimensión mayor cuando se relacionan con la llamada sociedad de la información, en la cual, la web es un medio que crea condiciones para un acceso libre al gran acervo de información y conocimiento disponible a través de los sitios conectados a ella. El acceso al conocimiento supone que lleva implícito el uso, las habilidades y la toma de decisiones desde las más sencillas hasta las más complejas, esto puede quedar en eso, un supuesto, porque la realidad puede ser diferente dependiendo de condiciones particulares de cada estudiante (por ejemplo socioeconómicas) y no lograrse los propósitos esperados.

Los niveles de presencia de las TIC en la educación variarán dependiendo del país del que se trate, de su nivel de desarrollo socio-económico y las costumbres culturales. Finalmente es un fenómeno social que se mide de acuerdo a manifestaciones vinculadas a las propias posibilidades, así como de su impacto, en donde los datos cuantitativos no necesariamente son un reflejo real sobre el aprovechamiento de las TIC. En educación, el análisis debe ser más profundo, relacionado con los beneficios tangibles, principalmente proyectados en el aprendizaje (UNDP, 2001).

La educación es el detonante principal de las manifestaciones del desarrollo social, aunque existen otros factores que pudieran considerarse una consecuencia, tales como: el acercamiento a idiomas diferentes a los propios (especialmente el inglés), la infraestructura del país, la cultura, los valores sociales y la calidad en la educación misma, todos vinculados de forma directa a la presencia de las TIC como elemento que moviliza e influye en muchos aspectos, contribuyendo a la eficiencia de los procesos y especialmente agilizando la divulgación del conocimiento, permitiendo así llegar de forma rápida a mayor número de personas.

México como país no puede estar exento de las observaciones hacia su grado de pertenencia a la sociedad del conocimiento, a la identificación de niveles de brecha digital y a la determinación de su infraestructura tecnológica como base del desarrollo en general. La definición de proyectos de desarrollo nacional en relación con las TIC tienen como propósito principal la reducción de la brecha digital, lo cual representa desarrollar planeaciones precisas con una visión integral, que contemple todos los niveles sociales y económicos de la sociedad dentro de un marco referencial del desarrollo nacional, aspecto que al no suceder, genera la desigualdad interna en acceso y en distribución social del conocimiento (Lau y Tarango, 2009).

Estudiar las condiciones en niveles de brecha digital que observa en una sociedad en general es factible y positivo, sin embargo, cabe considerar la importancia de de hacerlo desde la perspectiva de los sistemas educativos, los cuales tienen la ventaja de representar un medio estructurado y controlado para la promoción de todo 
conocimiento y cultura universal, en donde las acciones deberán estar basadas en la delimitación de políticas nacionales que fortalezcan la infraestructura, generen medios de difusión el acceso a la información, propiciando de una manera forma la generación del conocimiento, en este caso, en todos los aspectos relacionados con las TIC.

En el caso preciso del presente trabajo, el cual se considera una investigación empírica, debe de tomarse en cuenta que los resultados que se arrojan son de carácter diagnóstico exploratorio, propiciando la identificación de condiciones temporales sobre la presencia y ausencia de determinado elemento, en este caso las TIC, de las cuales se pueden derivar acciones concretas, tales como la necesaria consideración inmediata sobre los aspectos de capacitación de los estudiantes como usuarios, así como el arranque o continuidad de estudios diagnósticos sobre vertientes que afecten a directivos, personal académico y padres de familia en aspectos específicos tales como: niveles de acceso a las TIC (disponibilidad de equipo y conexiones a internet), habilidades en el uso de las tecnologías, la adquisición de la capacidad de identificar la relevancia de los contenidos y la relación de las acciones de acuerdo a las necesidades de aprendizaje propias de los niveles educativos y de los propósitos particulares en relación con necesidades de información y TIC.

\section{Educación y TIC: binomio de trabajo académico}

La difusión masiva del uso de las TIC ha transformado en mayor o menor medida los ámbitos sociales y económicos de todos los países del mundo. Esto ha provocado el surgimiento de una nueva dimensión del sistema económico internacional denominada globalización, el cual es un término que supera la simple concepción económica, representando también la interconexión de personas, sociedades y el crecimiento de las propias organizaciones, de tal forma que los rasgos de comportamiento tengan como característica la internacionalización del conocimiento (UNESCO, 2009).

El conocimiento es la base de los sistemas educativos y en las organizaciones ha llegado a sustituir las materias primas en orden de prioridad, además, al relacionarlo con las TIC, se convierte en un recurso estratégico en la generación y divulgación de nuevos conocimientos. Tal parece que la perspectiva de involucrar las tecnologías propiciará resultados significativos en los procesos educativos, pero la situación se complica cuando no existe o es limitada, propiciando la acentuación de la marginación social y de conocimiento, generando precisamente la llamada brecha digital.

En el marco de la globalización es conveniente identificar el lugar que ocupa México en relación con otros países respecto al nivel de penetración en las TIC, lo cual se ha considerado como elemento estratégico como parte del desarrollo. Por ejemplo, es importante visualizar los niveles de telefonía y su repercusión en el acceso a internet, la telefonía móvil, la disponibilidad de equipos de cómputo, etc. En muchos de estos indicadores México ocupa una mejor posición dentro de América Latina, sin embargo sigue habiendo descompensación interna en relación a las proporciones de distribución dentro de su propia población (CEPAL, 2009). 
El reto en la reducción de la brecha digital no es sólo la intromisión que se haga al compararse en las condiciones nacionales internas, ni tampoco en relación con aquellas naciones que observan un nivel socioeconómico igual o inferior al propio, sino la condición que se observa en brecha digital en los países industrializados donde los niveles son drásticamente inferiores, por ejemplo, en el caso de Estados Unidos y Canadá que han alcanzado niveles altos de disponibilidad de computadoras en la población, igualmente en servicios telefónicos y en el resto de los indicadores relacionados con las TIC. Aunque México puede observar niveles bajos de disponibilidad de TIC en la población en general, especialmente en comparación con los países antes mencionados, sorprende su crecimiento en los últimos años, además de los esfuerzos que han hecho los gobiernos por establecer políticas públicas al respecto.

Los indicadores anteriores se enfocan principalmente al registro de la disponibilidad y acceso de TIC en la población en general, sin embargo, y tomando como referencia el papel de la educación como sistema formal para el desarrollo de capital humano, existe la necesidad de abordar los temas de brecha digital desde una visión integral en donde se consideren además aspectos relacionados con el uso adecuado, la generación de conocimiento y la capacidad de evaluación y discriminación de los recursos informativos disponibles, todo ello con el enfoque de enfrentar los nuevos retos frente a la sociedad del conocimiento (Romo, Tarango, Murguía y Ascencio Baca, 2012).

Las transformaciones sociales del mundo son cada vez más rápidas, mostrándose como consecuencia un cambio en los aspectos económicos y culturales, aspectos que provocan nuevas formas de vivir de los ciudadanos. Una de las macrotendencias más significativa en tiempos actuales es sin duda la disposición de las TIC, caracterizado por el incremento, el ritmo de creación, acumulación, distribución y aprovechamiento de la información y el conocimiento, cambiando con ello las formas como los seres humanos se relacionan, propiciando incluso mayores grados de marginación (Lau y Tarango, 2012).

La brecha digital suele tener múltiples aristas, siendo el relacionado con procesos educativos uno de los más precisos al poderse medir a través de los niveles de alfabetismo digital, el cual es definido sobre las habilidades y competencias de los sujetos en relación con el acceso, uso y manejo de las TIC, inherentes a sus procesos o actividades académicas o laborales para solucionar problemas cotidianos. Este aspecto amerita además abordar la problemática desde la perspectiva de las distintas generaciones en la población, habiendo aparente diferencia en el comportamiento de los grupos sociales en relación con las TIC dependiendo de la época en que nacieron las personas, identificándose aquellos que nacieron antes del surgimiento de las tecnologías y los que ven este fenómeno como algo que ya existía en la sociedad y su adaptación suele ser mayor, llegando incluso a aparecer las concepciones de e-learning (aprendizaje electrónico), n-learning (aprendizaje nómada) o b-learning (blended learning o aprendizaje combinado), los cuales han llegado a ámbitos diversos de aplicación visualizando la predisposición a la movilidad casi natural de los sujetos (Attewell, 2005; Tarango y Marzal, 2011; Bruns, Cobcroft, Smith y Towers, 2007).

Medir la relación de los sujetos respecto a las TIC puede ser variado, por ejemplo, cuando se quiere desarrollar en todos los sectores de la población (López Noreña, 
2010), lo cual deriva múltiples ventajas al conocer la condición observada en un país, región, etc., sirviendo para la toma de decisiones y el establecimiento de políticas públicas nacionales, en relación a estrategias para mejorar las condiciones globales o identificar las formas de distribución de los beneficiarios de los servicios. Otra vertiente es cuando los procesos de evaluación comprenden a grupos o sistemas cerrados, en donde el control de los datos es más específico y su repercusión sería incluso delimitada a propósitos particulares de organizaciones y grupos específicos.

Aunque una de las formas más amplias de evaluar los niveles de brecha digital o de acceso a las TIC es la relacionada con los aspectos globales, o sea la que se presenta entre países, existen otras dos vertientes: la brecha social (referida al interior de una nación) y la brecha democrática, enfocada a grupos particulares de un estudio (Casado Ortiz, 2012). Independientemente de la dimensión de la evaluación de la brecha digital, pueden desarrollarse además análisis socio-demográficos específicos, tales como género, edad, nivel de ingresos y niveles educativos en los que están inmersos.

Las propuestas de estudio sobre brecha digital anteriormente descritos suelen pueden estar enfocadas a observar mayor énfasis en aspectos cuantitativos. Cuando se analizan aspectos como la calidad de los accesos, la disponibilidad de conexión de banda ancha o la aplicación de los contenidos para resolver problemas concretos, se dimensiona de forma distinta el acceso a las TIC. La Asociación Latinoamericana de Integración (2003) identifica cuatro dimensiones cualitativas: 1) motivación para acceder; 2) el acceso a determinado tipo de material; 3) las competencias para el acceso y 4) el acceso para usos avanzados. Además de lo anterior, y considerando los constantes cambios tecnológicos, un aspecto mayor de evaluar es la apropiación hacia las nuevas propuestas.

De acuerdo a la Cumbre Mundial sobre la Sociedad de la Información se ha enfatizado la función de las nuevas tecnologías como herramienta de comunicación de valores comunes entre grupos, individuos y organizaciones sociales, en donde se considera a las TIC como un fin más que como un medio para impulsar el cambio social (UNESCO, 2005). Esta clase de iniciativas no hace diferencias individuales ni grupales de toda índole, comprendiendo esto la llamada inclusión social.

Los beneficios sociales deben verse para comunidades y organizaciones, en donde la eficacia de las políticas de inclusión digital dependerá de las posibilidades de integración del conocimiento, por tanto, dependiendo de diversas condiciones sucederán distintos rasgos de comportamiento en los grupos evaluados, algunas veces suceden los acontecimientos producto de la eficacia de las políticas de inclusión social o bien sin ellas, ya sea por iniciativa de los miembros de los grupos o por necesidades individuales o comunes que provocan acciones homogéneas (CEPAL, 2009).

La difusión masiva de las TIC ha redefinido múltiples aspectos de la forma como suceden los procesos educativos, además ha contribuido a la creación de nuevos términos y conceptos, siendo uno de ellos el de brecha digital. Aunque este término suele tener múltiples aplicaciones, como el comercio electrónico y gobierno electrónico, siendo el enfoque de la sociedad de la información la que mejor define su relación con la educación, considerando que en esta última se define el perfil del 
ciudadano del futuro y es en los ámbitos educativos en donde mejor se puede influir, al menos de manera formal y controlada.

La inmersión de los sujetos en procesos formales de educación propicia tener acceso a información formal, en donde es posible recibir entrenamiento para usarla y saber usarla, además de que le permite la capacidad de toma de decisiones, esto representaría inmiscuirse, a la par que se atienden las cuestiones curriculares, acceder a mecanismos de alfabetización informacional digital. Esta situación es una inferencia que no necesariamente tiene validez, ya que los resultados varían dependiendo de los contextos en que suceden, especialmente vinculados a los niveles de desarrollo y costumbres culturales de los involucrados (Rival Oyarzún, 2010).

Entidades como la Fundación Santillana (2012) a través del desarrollo de un evento académico específico sobre brecha digital y educación, considera que la forma como llegan las TIC a los sujetos determina los propósitos que tendrán su uso y aplicación, esto significa que pudo haber tenido como intención aprender el uso de las tecnologías, aplicarla a la solución de problemas en los ámbitos laborales o suportar acciones académicas. Otro aspecto importante en el caso específico de las escuelas, es el hecho de quién proporciona los equipos y servicios de cómputo: las instituciones educativas o los estudiantes de forma independiente. En México existen indicios que la mayor parte de las tecnologías y el acercamiento a ellos ha sido por iniciativa propia de los estudiantes más que de las propias escuelas.

Las cuestiones del comportamiento que pueden observar los docentes y los estudiantes en las escuelas en relación a la importancia que se da al uso de la información formal resulta un aspecto de sumo interés a los propósitos de esta clase de estudios (Prensky, 2001; Jimmy, 2005). Se parte primero de las condiciones de la infraestructura disponible además de las habilidades de uso y la identificación de información relevante a los contenidos escolares, especialmente en el idioma y contexto de los usuarios. Todos estos aspectos al estar cubiertos debidamente ayudarán a la formación de los sujetos y se proyectarán en la toma de decisiones. De todos estos aspectos, las cuestiones de cambio cultural en los sujetos en identificar la importancia de las TIC resulta ser un proceso más costoso que la mera adquisición de infraestructura (Zárate Díaz, 2009).

\section{Planteamiento del problema}

Una de las preocupaciones principales del sistema educativo mexicano es elevar la calidad de la educación, habiendo la necesidad de investigar y proponer alternativas que permitan abatir el rezago educativo en todos los aspectos que dentro de este se propicien (académico, tecnológico, de cobertura, etc.), situación que si no se atiende es probable que se convierta en más grave, ya que tales problemáticas se aceleran más tomando como referencia la evolución constante que observa el desarrollo de las TIC.

Por lo tanto, el primer paso en el abordaje de cualquier situación relacionada con procesos educativos, que no se ha atendido en México, deberá partir de cuestiones diagnósticas, esto con el fin de dimensionar la situación y a partir de ello determinar la 
implementación de estrategias que permitan contribuir a superar el rezago a través de nuevas formas de trabajo, que propicien la interacción efectiva de los estudiantes, docentes, autoridades educativas y sociedad en general, esta vez bajo la propuesta de la integración de las TIC dentro del salón de clases, en los hogares y en los espacios públicos con el fin de contribuir a la mejor pertenencia a las exigencias de la sociedad del conocimiento.

La situación inicia su problematización en la medida que se desconoce el grado y forma de relación que tienen los estudiantes de la educación básica (educación secundaria, que en México comprende tres años una vez terminado seis que incluye la educación primaria) con las TIC, esto determinado, que hoy día, el conocimiento de las tecnologías están generalizados en la población y que es determinante tal interacción basada en la disposición que se tiene de tales elementos en el entorno del sujeto. Una preocupación fundamental es que las escuelas secundarias públicas mexicanas no tienen definido políticas formales para generar infraestructura informática de esta naturaleza, sin embargo se infiere que el origen del uso de las TIC surge de la iniciativa de los estudiantes por tener un acercamiento a ellas.

Existe una preocupación manifiesta en el interés de diversos actores de la educación básica mexicana en incorporar estrategias didácticas formales basadas en el uso de las TIC, lo cual está determinado en la identificación de diversos elementos vinculados al tema: disponibilidad de TIC, habilidades de acceso y uso de los recursos, niveles de conocimiento y entornos en los que sucede el acceso a las nuevas tecnologías. Tales condiciones determinarán la toma de decisiones en cuanto a la integración de acciones concretas para contribuir al desarrollo educativo equitativo, sin propiciar elementos de generación de falta de una inclusión social correcta.

\section{Justificación}

Un estudio de esta naturaleza se justifica en diversos aspectos fundamentales:

a). La difusión masiva del uso de las TIC, las cuales contribuyen a la transformación de ámbitos sociales y económicos, situación que al no ser atendida oportunamente propicia un rezago educativo basado en las limitantes del aprendizaje relacionado con los aspectos de la globalización.

b). Las exigencias de la llamada sociedad del conocimiento muestran alto vínculo con la capacidad del sujeto en relación con las TIC, en donde, al no existir una estrategia formal y eficiente de apoyo al desarrollo de habilidades en su manejo, se manifiestan limitantes en la educación de los involucrados, especialmente en la educación pública de México, la cual atiende a todos los sectores sociales.

c). El estudio de presencia y tipo de uso de las TIC permite la identificación de condiciones reales, mismas que a su vez propician identificar un potencial para promover el desarrollo académico, ya que se considera al fenómeno de las TIC como 
uno de los cambios paradigmáticos más importantes en la educación de los últimos tiempos.

d). El estudio de las TIC en la educación requiere de un análisis que tome en cuenta diversas condiciones propias de cada entorno, siendo la primera instancia la identificación de indicadores de disponibilidad o posesión de infraestructura, diferenciando la personal y la institucional.

\section{Objetivos de la investigación}

Este estudio propone los siguientes objetivos de investigación:

a). Determinar, de manera descriptiva, la separación digital que observan estudiantes de escuelas secundarias públicas de la Ciudad de Chihuahua, México.

b). Identificar el tipo de relación que existe con las TIC en diferentes escenarios posibles (escuela, hogar o entorno social).

c). Proyectar estrategias viables en la identificación de procesos de enseñanzaaprendizaje y alfabetización digital inmersos en el curriculum.

\section{Metodología}

La investigación está centrada en un enfoque cuantitativo ya que describe las ideas esenciales sobre las observaciones vertidas por los sujetos participantes basados en sus propias situaciones, por lo que corresponde a un análisis empírico. Las instituciones educativas seleccionadas fueron identificadas de acuerdo a las siguientes características homogéneas: 1) estar ubicadas en un sector de nivel socioeconómico medio, 2) observar una urbanización con todos los servicios básicos (luz eléctrica, agua, drenaje, pavimentación, etc.), 3) Ser escuela de dos turnos (matutino y vespertino) y 4) estar consideradas dentro del rango de grandes, las cuales tienen población suficiente con al menos cinco grupos por nivel por turno.

Una vez identificadas las tres escuelas a participar, se desarrolló un muestreo tipo probabilístico y aleatorio, la cual fue estratificada por el grado escolar cursado. Validando la muestra, la unidad de análisis fueron los alumnos pertenecientes a estas instituciones en estudio, sin ninguna restricción de edad, sexo, nivel socioeconómico o religión, o cualquier otro aspecto, salvo el grado escolar que cursan.

Para la definición de la muestra, al contar con una población promedio de 744 alumnos en los tres grados, la probabilidad de ocurrencia fue de $50 \%$ y la probabilidad de no ocurrencia fue de $50 \%$ y un error estándar del $7 \%$ con un nivel de confianza del 95\% dando, resultando un tamaño mínimo estadístico de 163 estudiantes. Sin embargo, la muestra real fue superior al mínimo, al lograrse participación de 187 estudiantes por institución, es decir un total de 561 estudiantes muestreados en total, aunque por la 
disposición para el desarrollo de la investigación, todos los datos fueron recolectados en el turno matutino de cada institución (tabla 1).

\begin{tabular}{ccccc}
\hline Grado & $\begin{array}{c}\text { Número de } \\
\text { estudiantes }\end{array}$ & $\begin{array}{c}\text { Tamaño de la } \\
\text { muestra }\end{array}$ & $\begin{array}{c}\text { Carga } \\
\text { muestral }\end{array}$ & $\begin{array}{c}\text { Muestra } \\
\text { real }\end{array}$ \\
\hline $1^{\circ}$ grado & 274 & 60 & 36,83 & 68 \\
$2^{\circ}$ grado & 247 & 54 & 33,20 & 62 \\
$3^{\circ}$ grado & 223 & 49 & 29,97 & 57 \\
\hline Total por & 744 & 163 & $100 \%$ & 187 \\
muestra & & & & \\
\hline
\end{tabular}

Tabla 1. Resultados de la muestra aleatoria estratificada

Una vez identificadas las instituciones educativas y las muestras de estudio, se procedió a la elaboración de un instrumento tipo encuesta como técnica para la recolección de datos. Esta encuesta incluye diversos apartados: uno referente a información personal de carácter anónimo, registrándose la edad, género, tipo de vivienda y escolaridad de los padres y el resto, compuesto por 18 reactivos (16 de respuesta cerrada y dos de respuesta abierta), siendo sus indicadores base de evaluación respecto a la dimensión de la brecha digital (identificada por la OCDE a nivel internacional y por INEGI a nivel nacional, 2003) los siguientes: conceptualización de las TIC, identificación del equipo de cómputo, Internet, televisión, telefonía fija, banda ancha, habilidades de uso y características de la institución educativa en relación con entornos relacionados con la tecnología (anexo 1).

Los principales elementos de marco conceptual que comprende este estudio son: brecha digital, TIC, internet, telefonía móvil, banda ancha y escuela pública, mismas que no se definen aquí ya que resultan ser conceptos de dominio generalizado, salvo las especificaciones pertinentes que se han incluido dentro del documento general.

La forma de aplicación del instrumento fue de manera directa, agrupando a los sujetos por grado académico en un espacio para la recolección de datos. Dadas las condiciones de edad y nivel académico de los sujetos participantes fue necesario ir leyendo cada una de las preguntas para su respuesta, haciendo las explicaciones pertinentes en cada caso.

\section{Marco contextual: caracterización del estudio del caso}

Las tres instituciones educativas seleccionadas corresponden a las características antes descritas referentes a su tamaño, condición socio-económica, tamaño y forma de operación. Además de la disponibilidad institucional para desarrollar el estudio. Puede considerarse que las tres instancias seleccionadas corresponden a las típicas instituciones educativas de nivel básico (secundario) ubicadas en ciudades de tamaño medio de México (de 800,000 a un millón de habitantes), con las limitantes de infraestructura que caracterizan a esta clase de instituciones. 
Las características a nivel general y particular del caso de estudio en donde tuvo lugar el desarrollo de la investigación corresponden, en principio, a dimensionar aspectos relacionados con las características del personal promedio que integran a las instituciones involucradas. El personal administrativo se constituye de nueve personas entre personal directivo y operativo con un promedio de antigüedad en el servicio de 11,6 años y en la institución de 7,62 años, así como de edad de 37,7 años. El personal directivo cuenta con grado de maestría en educación. Respecto al personal docente, cada institución se compone de 30 docentes; $46,67 \%$ tienen grado de maestría, 43,33\% grado de licenciatura (ambos con una variedad de especialidades prevaleciendo el área de educación, administración de empresas, educación física e ingeniería industrial) y el $10 \%$ de nivel de preparatoria, con 8,67 años promedio de servicio en educación y 5,7 años promedio en la institución.

\section{Análisis de resultados}

Las instituciones educativas participantes tienen grupos en grados de primero y segundo y tercero, en promedio cinco grupos por nivel. El promedio de estudiantes por institución es de 744, de los cuales, según la muestra evaluada, el $46,51 \%$ son mujeres y $43,49 \%$ hombres, el promedio de estudiantes por grupo es de 50 . También, de las muestras evaluadas, $93.58 \%$ manifestó vivir en casa propia (pagada o hipotecada) y el $6,42 \%$ rentada.

Respecto a los padres de familia de los estudiantes, se observó que el $68,98 \%$ de los mismos poseen estudios de nivel superior, $18,71 \%$ estudiaron hasta el nivel de preparatoria o nivel técnico, $10,16 \%$ únicamente estudiaron hasta el nivel de secundaria y sólo el 2,15\% nivel básico de educación primaria. Este dato resulta interesante en el sentido de que la mayoría de los padres de familia tuvieron acceso a educación profesional, lo cual generalmente los involucra en empleos que demandan el uso de las TIC o se tiene conocimiento de ellas.

El punto de partida en la identificación de la conceptualización que tengan los sujetos en observación respecto a lo que identifican como TIC, para lo cual se proporcionaron tres elementos básicos: telefonía móvil, banda ancha e Internet, de lo cual se observó que el $41,71 \%$ identificó los tres tipos antes expuestos, el $56.14 \%$ consideró únicamente al Internet, 1,60\% determinó que el teléfono móvil se incluía en este rubro y sólo $.53 \%$ identificó a la banca ancha.

Un aspecto importante en la identificación de los niveles de brecha digital es determinar si los sujetos en evaluación tienen acceso a las TIC al contar con equipo de cómputo en su hogar, sin importar ahora el grado de obsolescencia que este observa, cuya respuesta fue que el $98,39 \%$ de los encuestados poseen equipo de cómputo en su hogar y sólo el 1,61\% manifestó no poseerlo. Tal resultado pone de manifiesto un hallazgo importante, en el sentido de la amplia posesión de equipo dentro de los hogares, lo cual acerca más al sujeto a su uso cotidiano.

Del total de estudiantes participantes en la muestra que manifestaron posesión de equipo de cómputo dentro de su hogar, sorprende que la totalidad han manifestado 
compartirlo con algún miembro de la familia, ya sea con los hermanos, con alguno de los padres de familia o con toda la familia, lo que pone de manifiesto que, al menos, dos miembros de la familia tienen acceso a las TIC de forma regular.

Un aspecto importante, más allá de la mera posesión de equipo de cómputo dentro del hogar, es el hecho de considerar su nivel de obsolescencia o vejez de los equipos, en donde, de acuerdo a los datos recolectados en las muestras de estudiantes que poseen equipo de cómputo en su hogar, es el siguiente: el 70,05\% tiene equipo con un máximo de obsolescencia de dos años; el $21,92 \%$ con una antigüedad de tres a cinco años; el 4,27\% poseen equipo de seis a ocho años de antigüedad; y sólo, el 3,74\% poseen equipo con un nivel de obsolescencia superior a los nueve años. Los resultados denotan que la mayoría de los encuestados poseen equipos modernos y las minorías equipos con cierto grado de obsolescencia, considerando que las nuevas generaciones de equipo de cómputo se renuevan cada seis meses, sin embargo, con los grados de obsolescencia observado siguen siendo funcionales para los propósitos académicos que las instituciones educativas demandan y en la conectividad con otros servicios de información.

Ante el cuestionamiento sobre la forma como se define Internet, considerando que este aspecto es esencial para abatir la brecha digital ya que a través de él se tiene acceso al conocimiento universal, los estudiantes expresaron de forma abierta su propia concepción, para lo cual, uniformando lenguaje expresado se concentra en tres expresiones básicas: una herramienta de investigación (47,59\%); una red internacional pública para acceder a la información (47,59\%); una red privada $(0,05 \%)$ y 4,27\% manifestaron no saber definirlo.

Otra pregunta abierta a los estudiantes participantes en la muestra, fue en relación con el uso que es posible dar al Internet como herramienta de trabajo de toda índole, observándose que: $46,73 \%$ consideran que es viable para desarrollar operaciones comerciales al adquirir productos; $21,73 \%$ lo aplicarían para acceder a información virtual con fines académicos; $9,78 \%$ para desarrollar trámites gubernamentales y el $21,76 \%$ consideran los tres anteriores.

En relación con el medio de comunicación que manifestaron utilizar los estudiantes en evaluación para conectarse a Internet, el resultado observado es que: el $72,72 \%$ utilizan los servicios de telefonía fija y móvil, 22,99\% utilizan el servicio de telecable, $4,79 \%$ no tienen conexión y ninguno de los encuestados usan banda ancha.

El análisis anterior centra su atención en la presencia de las TIC y sus servicios de información en el conocimiento del sujeto y en la presencia dentro del hogar, sin embargo, en cuanto a la identificación de esta clase de infraestructura dentro de las escuelas evaluadas, el resultado recolectado demuestra que la totalidad de los estudiantes tienen plenamente identificado que su institución no cuenta con un centro de cómputo para su uso.

Si bien el uso del Internet tiene gran importancia en los estudios de brecha digital, la frecuencia en su uso con fines específicos agrega valor al análisis de los datos. Para este caso, se analiza la frecuencia de uso del Internet con fines académicos, 
observándose que: el 78,6\% lo usan algunas veces, sólo el 8,07\% lo utilizan siempre con ese fin, $6,41 \%$ lo usan rada vez y el $6,92 \%$ nunca lo usan.

Respecto a la presencia de Internet libre dentro de la escuela, considerando la posibilidad de que las instituciones tengan contratado algún servicio o bien a través de la señal de algún servicio dispuesto en general para la sociedad, las respuestas estuvieron divididas en cuanto a qué: $22,45 \%$ dijo que siempre, $26,73 \%$ respondieron que algunas veces, $20,85 \%$ indicaron que ocasionalmente, mientras que el $29,97 \%$ respondieron que nunca.

En relación al nivel de dominio que los estudiantes en evaluación tienen sobre el uso del Internet, las respuestas fueron positivas ya que, de acuerdo a sus percepciones, el $82,5 \%$ consideró que era bueno, sólo el $12 \%$ regular y 5,5\% malo. Es importante indicar que las respuestas recolectadas fueron de toda la muestra y se incluye aquellos estudiantes que no poseen equipo de cómputo y formas de acceso dentro de sus hogares.

Referente con el Internet, un medio frecuente utilizado como forma de comunicación es el correo electrónico. Ante la pregunta, si los estudiantes evaluados utilizan este medio para desarrollar actividades académicas de la escuela, los resultados obtenidos fueron: $59,35 \%$ lo usan algunas veces o poco, $39,57 \%$ no lo utilizan y sólo $1,06 \%$ manifestó usarlo siempre con fines académicos. Ciertamente, todos los alumnos en evaluación utilizan el correo electrónico, sin embargo, su enfoque a fines académicos se ve reducido ampliamente.

La telefonía móvil como recurso para el aprovechamiento escolar de los estudiantes como resultado que del total de las muestras evaluadas, muestra que el 95,18\% de los estudiantes poseen un teléfono móvil activo y sólo el 4,82\% no lo poseen. Del porcentaje de estudiantes que poseen teléfono móvil activo, se identificó que este medio es poco utilizado con fines académicos, ya que el $42,24 \%$ de los encuestados manifestaron que nunca lo utilizaban con ese fin, el $24,06 \%$ rara vez, el $20,32 \%$ algunas veces y sólo el $13,38 \%$ lo utilizaban siempre con fines de resolver acciones relacionadas con sus actividades de la escuela.

Un fenómeno interesante es el que se manifiesta al preguntar a los estudiantes involucrados en el estudio que poseen teléfono móvil si éste lo utilizan para conectarse a Internet, las respuestas fueron contrarias en frecuencia al uso con fines académicos, el $60,96 \%$ dijeron que siempre lo utilizan con fines de conexión a internet, el 20,32\% alunas veces, $3,73 \%$ indicaron que rara vez y el $14,99 \%$ que nunca.

Otro aspecto fundamental en el estudio de la brecha digital es la viabilidad de uso de las TIC como forma alternativa para el desarrollo de actividades académicas, la perspectiva de los estudiantes en evaluación fue que el 56,14\% manifestaron reconocer el Internet con ese fin, $1.60 \%$ consideró a la telefonía móvil, $0,55 \%$ a banda ancha y $41,71 \%$ indicaron que todas las anteriores. Esta cuestión ofrece diversas pautas sobre posibles potencialidades en el uso de las TIC con fines académicos.

Además, la consideración de la televisión como elemento para medir los niveles de brecha digital es evaluada en este estudio a través de la identificación del medio por el 
cual los estudiantes que participaron en la investigación acceden a ella. Las respuestas recolectadas manifiestan que todos tienen acceso a la televisión, sólo que el 96,79\% lo hacen a través de televisión de paga y el 3,21\% a través de televisión abierta.

Finalmente, se cuestionó a los estudiantes en evaluación sobre su opinión si las TIC tendrán una influencia personal que se manifieste como aprendizaje para la vida, esto con el fin de valorar la importancia de esta clase de herramientas en el futuro del estudiante en otros niveles o en su vida laboral, de lo cual se observó que: $88,23 \%$ definió que siempre le serviría este conocimiento, 3,74\% opinó que algunas veces o poco y el 8,03\% consideró que no tenía utilidad alguna en su vida futura.

Una vez obtenidos los anteriores resultados, se presentaron los resultados a los docentes y autoridades de las instituciones participantes a través de sesiones de grupo, en donde se consideraron los siguientes aspectos:

a). Existe una brecha digital muy corta en los sujetos de estudio y las TIC, sin embargo están más centradas en la posesión y uso indeterminado de las mismas para resolver situaciones generales (sociales o de diversión) más que académicas.

b). Se atribuye la alta presencia de TIC en el hogar, no sólo determinado por el nivel socio-económico de las familiar a las que pertenecen los sujetos estudiados, especialmente provocadas por los niveles educativos altos que observan la mayoría de los padres de familia.

c). Es necesario diagnosticar los niveles de alfabetización digital de los docentes de las instituciones educativas participantes, ya qué, se prevén algunas limitaciones en el uso de las TIC, específicamente para ser aplicadas a aspectos didácticos.

d). Se determina que la inclusión de las TIC dentro de las actividades académicas extraescolares cabe la posibilidad de suceder de forma voluntaria más no obligatoria, ya que los alumnos que no tienen posesión, acceso y uso de esta clase de tecnologías (que son pocos), no estarán obligados a participar en actividades académicas a través de ellas. De lo contrario sucederían problemas con padres de familia y con sus autoridades superiores.

e). Se considera, de forma importante, que la identificación de las TIC dentro del esquema de la actividad académica y personal de los sujetos en estudio, surgen de la iniciativa propia de la familia de los mismos, ya que la escuelas no tienen lineamientos formales ni recursos disponibles para el desarrollo de infraestructura que permita que el estudiante conviva académicamente con las nuevas tecnologías. De aplicarse a los procesos didácticos deberán suceder en eventos académicos extra-clase y no dentro de las actividades desarrolladas en el aula. 


\section{Conclusiones}

Al observar la alta disposición y acceso a las TIC por parte de estudiantes, puede concluirse que no existe una brecha digital marcada, sin embargo, si se analiza la forma o propósito de uso, puede decirse que existe una brecha digital marcada en relación con el desarrollo de actividades académicas. Tal situación se verá disminuida en la razón que las autoridades implanten políticas públicas de accesibilidad a la web, para que todos los estudiantes, independientemente de las características de su entorno puedan tener acceso a las tecnologías y sean usadas adecuadamente para mejorar los procesos de enseñanza-aprendizaje.

La necesidad de ofrecer programas de alfabetización digital, tanto a los estudiantes como a los docentes, directivos y padres de familia, les permitiría pasar del mero acceso y uso indeterminado en cuanto a sus propósitos, alcanzando la apropiación de las nuevas tecnologías, ya que estas pueden formar parte de los procesos de desarrollo académico, combatiendo incluso las demás brechas que subyacen a la digital.

Abordar la brecha digital únicamente a través de la mera posesión de TIC es considerado una limitante, vista desde la perspectiva educativa, ya que aunque es una de las formas como han establecido algunas políticas públicas mexicanas, incluso involucrado a entidades privadas, con fines de reducir este fenómeno realmente no se logra en la medida que las comunidades receptoras de estos procesos de equipamiento tecnológico poseen desconocimiento de uso adecuado, además de que los planes de estudio deberán establecer lineamientos formales de trabajo en relación a solucionar problemas vinculados con las TIC, tanto dentro del ámbito escolar o como actividades extraescolares.

Partiendo de los resultados obtenidos se rescatan aspectos valiosos en cuanto a que algunas dimensiones de la brecha digital están fortalecidas para que esta no sea notoria, especialmente en lo siguiente: identificación de las TIC (para uso académico presente y con utilidad futura), posesión y accesibilidad de equipo de cómputo dentro del ámbito del hogar, conocimiento suficiente del manejo de Internet en términos generales, disposición de otras TIC como le televisión de paga, la telefonía fija y móvil en todos los participantes. Además de la disponibilidad de los docentes y autoridades hacia la inclusión de las TIC en la enseñanza y el interés de incluir programas formales de alfabetización digital, además del alto nivel académico de los padres de familia.

Las debilidades más sobresalientes son: no existir parámetros académicos establecidos para el uso obligado de las TIC, usar las nuevas tecnologías sin un fin académico determinado regularmente vinculado a la mera diversión o convivencia social. Se considera que la mayor debilidad estriba en la falta de infraestructura y equipamiento de las instituciones educativas participantes en su carácter de públicas, lo cual limita el uso de las TIC, tanto por estudiantes como por profesores y restringiendo el desarrollo de acciones educativas relacionadas con las nuevas tecnologías dentro del ámbito escolar. 


\section{Referencias bibliográficas}

ASOCIACIÓN LATINOAMERICANA DE INTEGRACIÓN. (2003). La Brecha digital y sus repercusiones en los países miembros de la ALADI. (En línea). Consultado en: http://www.itu.int/wsis/newsroom/coverage/publications/docs/aladi_brecha_digitales.pdf.

ATTEWELL, J. (2005). Mobile technologies and learning: A technology update and m-learning project summary, Technology Enhanced Learning Research Centre, Learning and Skills Development Agency. (En línea). Consultado en: http://www.lsda.org.uk/files/PDF/041923RS.pdf.

BRUNS, A., COBCROFT, R., SMITH, J., TOWERS, S. (2007). Mobile Learning Technologies and the Move towards 'User-Led Education'. En: Proceedings Mobile Media, Sydney, Australia.

CASADO ORTIZ, R., Dir. (2012). Claves de la alfabetización digital. Madrid, España: Fundación Telefónica.

CEPAL. (2009). La sociedad de la información en América Latina y el Caribe: desarrollo de las tecnologías y tecnologías para el desarrollo. (En línea). Consultado de: http://www.oei.es/tic/cepal.pdf.

FUNDACIÓN SANTILLANA. (2012). Las Tecnologías de la información y la comunicación (TIC) en la educación: retos y posibilidades. (En línea). Consultado en:

http://www.fundacionsantillana.com/upload/ficheros/paginas/200906/xxii_semana monografica.pdf.

JIMMY, R. (2005). La Tecnología de la Información y la Comunicación (TIC). Su uso como Herramienta para el Fortalecimiento y el Desarrollo de la Educación Virtual. (En línea). en: http://www.cibersociedad.net/archivo/articulo.php?art=218.

LAU, J., TARANGO, J. (2009). Brecha más cognitiva que digital: papel de las habilidades informativas en países emergentes. En: Memorias de la II Conferencia Internacional sobre Brecha Digital e Inclusión Social. Getafe, Madrid, España: Universidad Carlos III de Madrid.

LAU, J., TARANGO, J. (2012). Academic libraries in México with an emphasis on the use of technology in Mexican state university libraries. En: Ravindra N. Sharma (Ed.). Libraries in the early $21^{\text {st }}$ century: an international perspective, vol. II. Berlín, Alemania; Boston, Mass.: Walter de Gruyter; IFLA.

LÓPEZ NOREÑA, G. (2010). Apuntes sobre cibercultura y la alfabetización digital posmoderna. (En línea). Consultado de: http://biblioteca.utec.edu.sv/siab/virtual/elibros_internet/55707.pdf. 
RIVAL OYARZÚN, H. (2010). Tecnologías de la información y comunicación en el sistema escolar chileno: aproximaciones a sus logros y proyecciones. Revista Iberoamericana de Educación, No. 51/2, 10 de enero.

PRENSKY, M. (2001). Nativos digitales, inmigrantes digitales. On the Horizon, Vol. 9, No. 6.

RAMENTOL, S. (2004). Teorías del desconcierto: viaje al fondo de la incertidumbre: los pensadores que diseñan el futuro global. Barcelona, España: Urano.

ROMO, J., TARANGO, J., MURGUÍA, P., ASCENCIO BACA, G. (2012). Cibercultura estudiantil en comunidades académicas de universidades públicas Mexicanas. Anales de Documentación, Vol. 15, No. 1.

TARANGO, J., MARZAL, M.A. (2011). Funcionalidad de la alfabetización informativa y sus ámbitos de actuación: aproximaciones a los entornos mexicanos y españoles. Revista Interamericana de Bibliotecología, Vol. 34, No. 3.

UNESCO. (2005). Cumbre mundial de la sociedad de la información. (En línea). Consultado en: http://www.itu.int/wsis/index-es.html.

UNESCO. (2009). Medición de las tecnologías de la información y la comunicación (TIC) en educación: manual del usuario. Montreal, Canadá: UNESCO, Instituto de Estadística.

UNDP. (2001). Human development report 2001: making new technologies work for human development. New York: Oxford University Press.

ZÁRATE DÍAZ, A. (2009). Los actores de la educación frente a las Tics. Aula Urbana, No. 74.

\section{Correspondencia con los autores:}

Javier TARANGO ORTIZ

José Refugio ROMO GONZÁLEZ;

Laura Patricia MURGUÍA JÁQUEZ;

Gerardo ASCENCIO BACA

Universidad Autónoma de Chihuahua, Facultad de Filosofía y Letras

Avenida Universidad s/n Ciudad Universitaria, Chihuahua, Chih., México 31170 e mail: tj.88888@hotmail.com;

Teléfono: (614) 4882304 


\section{ANEXO 1 \\ Encuesta de estudiantes}

Instrucciones: A continuación se incluyen una serie de preguntas, las cuales deberás contestar de forma clara y sincera en relación al conocimiento que posees del uso de las nuevas tecnologías de la información. Este cuestionario no afecta en ningún aspecto de tus calificaciones y tiene como fin conocer mejorar nuestras formas de trabajo dentro del aula.

\section{DATOS PERSONALES}

Edad

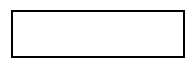

Género: Masculino

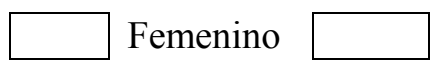

Su vivienda es: $\quad$ Propia $\square$ Rentada $\square$ Prestada $\square$

Nivel de estudios de los padres de familia:

Ninguno $\square$ Primaria $\square$ Secundaria

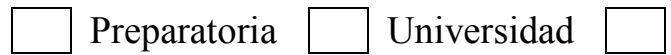

\section{USO Y MANEJO DE TECNOLOGÍAS}

1. Según tú entiendas ¿cuáles son consideradas tecnologías de la información y las comunicaciones o TIC? Marca o agrega las que consideres:
a). Teléfono móvil
c). Internet
b). Banda Ancha
d). Todas las anteriores

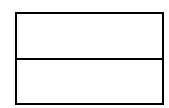

Otras:

2. ¿Posees uno o varios equipos de cómputo en tu casa?

SI
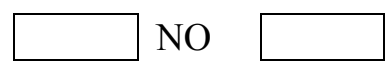

3. En caso de poseer equipo de cómputo en tu casa ¿con quién compartes el equipo de cómputo?
a). Con nadie
c). Con mis padres

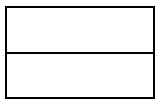
b). Con mis hermanos
d). Con toda la familia

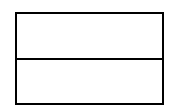

4. En caso de poseer equipo de cómputo en tu casa ¿cuántos años tiene tu equipo de cómputo? 

a). De 0 a 2 años
c). De 6 a 8 años

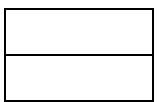
b). De 3 a 5 años
d). De 9 o más años

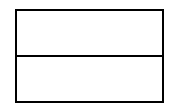

5. Define con tus propias palabras lo que entiendes por Internet:

6. ¿Cuál crees que es el uso más útil que le puedes dar al Internet?

7. ¿Cuál es la forma como te conectas?
a). Teléfono de casa
c). Banda Ancha
b). Teléfono móvil
d). No Tengo

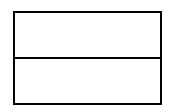

8. ¿Tu escuela cuenta con centro de cómputo?
a). $\mathrm{Si}$
c). está en construcción
b). No
d). Sí, pero no lo usamos

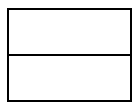

9. ¿Utilizas el Internet para desarrollar actividades académicas de la escuela?
a). Siempre
c). Rara vez
b). Algunas veces
d). Nunca

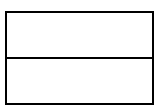

10. ¿Tienes acceso libre a Internet en tu escuela?
a). $\mathrm{Si}$
c). A veces

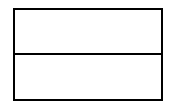

b). No

d). Lo ignoro

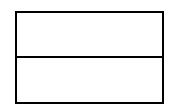

11. ¿Cuál es el nivel de dominio que consideras que tienes en el uso del Internet?
a). Bueno
c). Malo

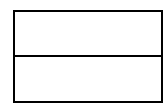

b). Regular

d). Nulo

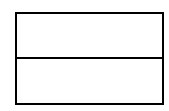


12. ¿Utilizas el correo electrónico para desarrollar actividades académicas de la escuela?
a). Siempre
c). Poco

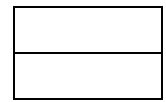
b). Algunas veces
d). Nada

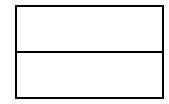

13. ¿Tienes tu propio teléfono móvil?

SI

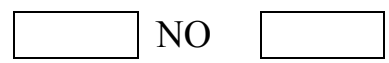

14. En caso de poseer teléfono móvil ¿lo utilizas para desarrollar actividades académicas de la escuela?
a). Siempre
c). Poco

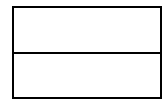

b). Algunas veces

d). Nada

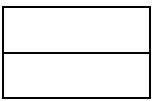

15. En caso de poseer teléfono móvil ¿lo utilizas para conectarte a Internet?
a). Siempre
c). Poco

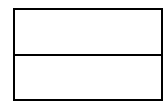

b). Algunas veces

d). Nada

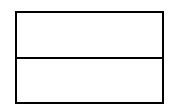

16. ¿Cuál de las TIC consideras viable para desarrollar tus actividades académicas de tu escuela?
a). Teléfono móvil
c). Internet

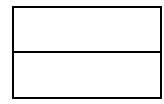
b). Banda Ancha
d). Todas las anteriores

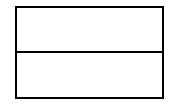

17. ¿Cuál es el medio donde ves televisión?
a). Tele Cable
c). Televisión abierta
b). Dish
d). Ninguno de los anteriores

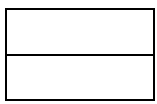

18. En tu opinión ¿las TIC pueden servirte durante toda la vida?
a). Siempre
c). Poco

b). Algunas veces

d). Nada

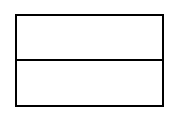

